\title{
A Case of Choroid Plexus Papilloma in the Lateral Ventricle, Complicated by a Neuroepithelial Cyst in the Posterior Fossa in a Newborn Infant
}

\author{
EIICHIRO HONDA, TAKASHI HAYASHI* AND SHINKEN KURAMOTO** \\ Department of Neurosurgery, Kitakyushu Chuo Hospital, Kitakyushu, 802, \\ *Department of Neurosurgery, St. Mary's Hospital, Kurume, 830 and \\ **Department of Neurosurgery, Kurume University School \\ of Medicine, Kurume, 830 Japan
}

Received for publication April 7, 1984

\begin{abstract}
Summary: The incidence of brain tumor in the newborn is extremely low. Choroid plexus papilloma of neuroectodermal origin has been reported in only nine cases, including the present case. Early diagnosis of brain tumor in the newborn is difficult. In addition, there are many difficult factors in the operative treatment of choroid plexus papilloma because of its tendency to hemorrhage and malignancy. Interestingly, a neuroepithelial cyst developed in the posterior fossa following the first operation. We report the present case as an extremely interesting one since the development of neuroepithelial cyst and choroid plexus papilloma appeared to have a common origin on the basis of histological findings, as well as other clinical observations.
\end{abstract}

Key words: neonatal brain tumor - choroid plexus papilloma - hydrocephalus - ventricular hemorrhage - neuroepithelial cyst

\section{Introduction}

Among infantile tumors, especially neonatal, few brain tumors have been reported. Of brain tumors in children under fifteen years of age, reported by Arnstein (1951), Jellinger (1973) and Sato (1978), only about $1.5 \%$ were neonatal.

Many of such patients die soon after their birth and extremely few cases survive, because of the high incidence of complication of congenital malformation, as well as physical weakness. In the present study, we succeeded in the overal removal of a giant choroid plexus papilloma, which had developed in the neonatal lateral ventricle, and also, in the excision of a neuroepithelial cyst, which first developed in the posterior fossa almost four months after the first operation. This is believed to be an interesting case where satisfactory progress was observed after operative treatment.

\section{Case}

Patient

boy (postnatal day 30)

\section{Family and case histories}

non-contributory. The pregnancy and delivery was normal.

\section{Present disease}

The mother had observed an enlargement of the cephalic circumference on and after postnatal day 20 and there was vom- 
iting, and weakened suckling ability, later rigidity of extremities and weak crying also became apparent, on and after postnatal day 30. Hydrocephalus having been diagnosed elsewhere, he was brought to our department and immediately hospitalized.

\section{Findings on admission}

Irregular respiration and anemia were observed systemically. As for the conscious state, slight crying in response to painful stimuli and poor voluntary movements were observed. The anterior fontanelle was enlarged and the tension was high. The light reflex was sluggish on the right side and anisocoria was observed (Right $>$ Left.) Neither retinal hemorrhage nor papilloedema was observed in the ocular fundus.

\section{Results of examinations}

General tests on the day of admission revealed the following:

WBC $13000 / \mathrm{mm}^{3}$, RBC $308 \times 10^{4} / \mathrm{mm}^{3}$, $\mathrm{Hb} 9.7 \mathrm{~g} / \mathrm{dl}$, hematocrit $30.0 \%$, Na 149 $\mathrm{mEq} / 1, \mathrm{~K} 3.7 \mathrm{mEq} / 1, \mathrm{Cl} 112 \mathrm{mEq} / 1$. Anemia was present. Plain CT scan revealed a high density mass in the pars trigon of the right lateral ventricle. The right lateral inferior horn was subjected to pressure collapse. The right frontal horn and contralateral ventricle were prominently enlarged. Bilateral drainage of the ventricles was performed after diagnosis of internal hydrocephalus due to an intraventricular tumor (Fig. 1). Table 1 shows the results fo examination of the cerebrospinal fluids (CSF). The left ventricular CSF showed a high protein content of $160 \mathrm{mg} / \mathrm{dl}$, while the right one exhibited a high blood con tamination. No communication was observed between the left and right ventricles. These findings suggested an obsturction of the foramen of Monro (Table 1).

\section{Operative findings}

Since the clinical symptoms were temporarily improved following ventricular drainage, radical operation was performed
TABLE 1

CSF findings from both ventricles

\begin{tabular}{ccc}
\hline & Rt. ventricle & Lt. ventricle \\
\hline CSF appearance & Bloody & slight \\
protein & - & $\chi$ anthocromic \\
cell count & $101 / 3$ & $160 \mathrm{mg} / \mathrm{dl}$ \\
Nonne-Apelt & $H$ & $5 / 3$ \\
pandy & $H$ & + \\
\hline
\end{tabular}

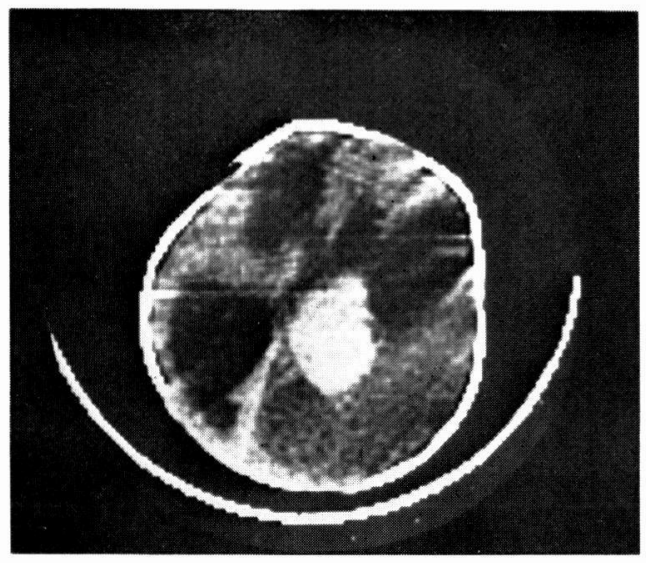

a

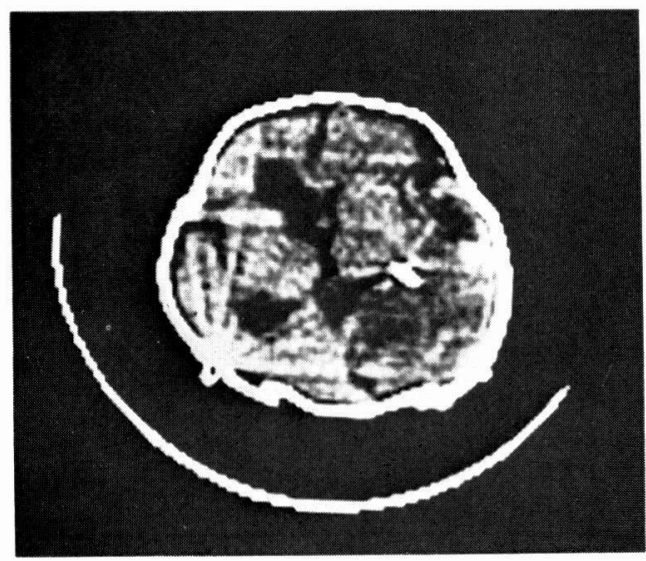

b

Fig. 1.

a) Plain CT showing high density tumor at the trigone associated with marked ventricular dilatation.

b) Two months after the removal of the tumor. There was no mass effect. A low density area remained at the trigone. 
ten days after admission.

Craniotomy extended from the right temporal part to the vertex. The surface of the brain was widely exposed and there was a brown, membranous appearance due to degeneration. Cerebrotomy was performed on this degenerated angular gyrus (Raimondi et al. 1975). The range of cerebrotomy was about $4 \mathrm{~cm}$ in length. There was free flowing and clotting blood just below this angular gyrus and a tumor was partially observed.

After removal of the clot and careful dissection, the whole of the tumor became apparent. This tumor, which was associated with multiple foci of hemorrhagic necrosis, was clearly distinguishable from its surroundings. After the tumor was removed, the main feeder artery (choroidal artery), which was buried at the bottom of the tumor, was clipped for hemostasis. The old clot in the ventricle was partially organized and occluded the right frontal horn and the homolateral foramen of Monro.

\section{Histological findings}

The tumor weighed about seventy grams and was grayish white to pink. The surface was quite smooth, although one part had a cauliflowerlike appearance (Fig. 2).

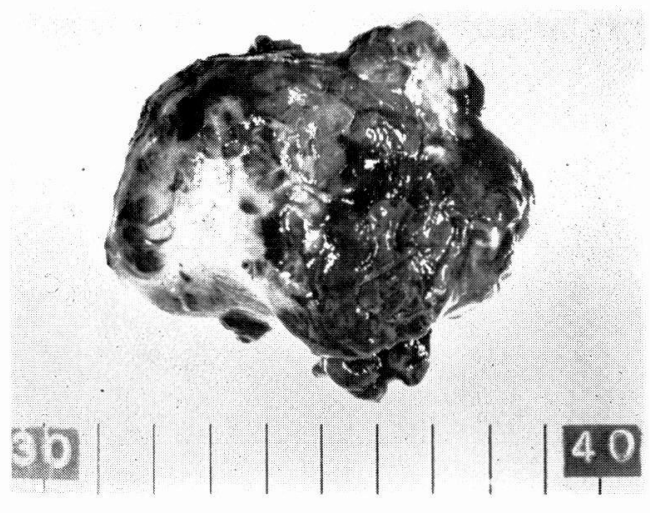

Fig. 2. Gross appearance of the tumor after removal. Multiple hemorrhagic foci of necrosis are seen on the surface of the tumor.

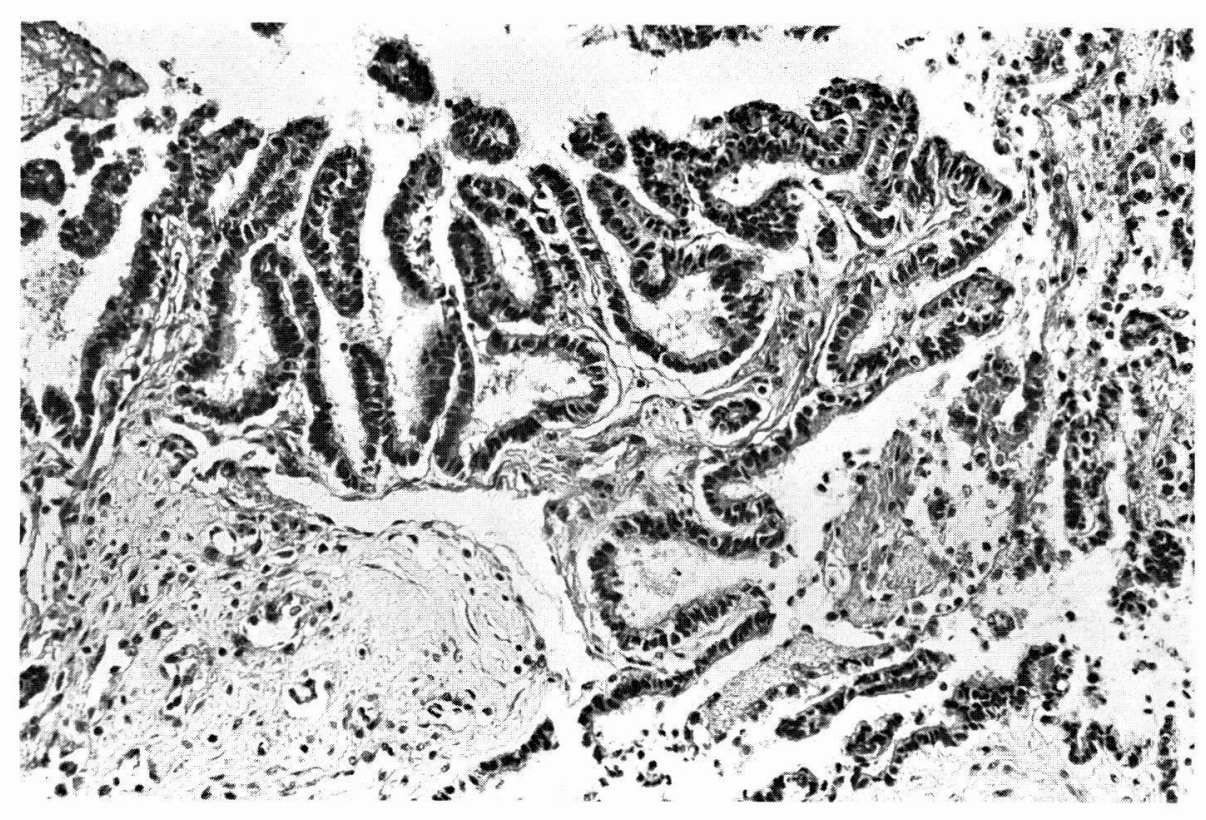

Fig. 3. Section of a typical choroid plexus papilloma. $\times 490$ Hematoxylin and eosin staining. 


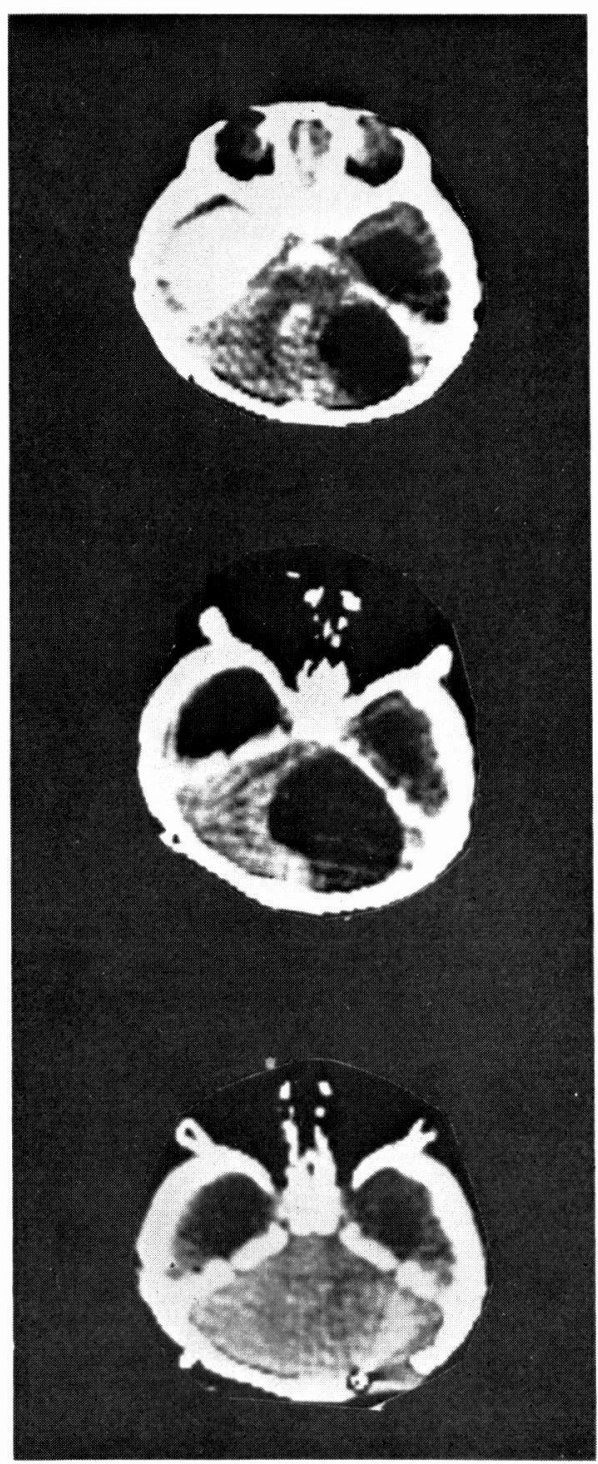

Fig. 4.

a) Metrizamide CT ventriculography. This showed a low density tumor at the right posterior fossa and shift of 4 th ventricle to left.

b) Enlargement of cystic tumor was showed on CT taken at 20 days after the initial CT.

c) 14 days after resection of tumor membrane. The cystic tumor had disappeared.
Scattered hemorrhagic necrosis was also observed on the cut surface. No calcification was observed macroscopically.

Histological examination showed a proliferating epithelial choroid plexus, which was arranged in papillary form indicating a typical choroid plexus papilloma. The intracellular nuclei were rich in chromatin. Multinuclear formation, heteroplasia or giant cells, however, were not observed. Malignancy of the tumor could, therefore, be rejected (Fig. 3).

\section{Postoperative progress}

Fifty days after the operation, only a disturbance of the right oculomotor nerve was observed. CT revealed only a low density area around the pars trigon of the right ventricle. The patient was, therefore, discharged (Fig. 4).

After a lapse of four months, the patient was hospitalized again, mainly because of weakened suckling ability and irritability.

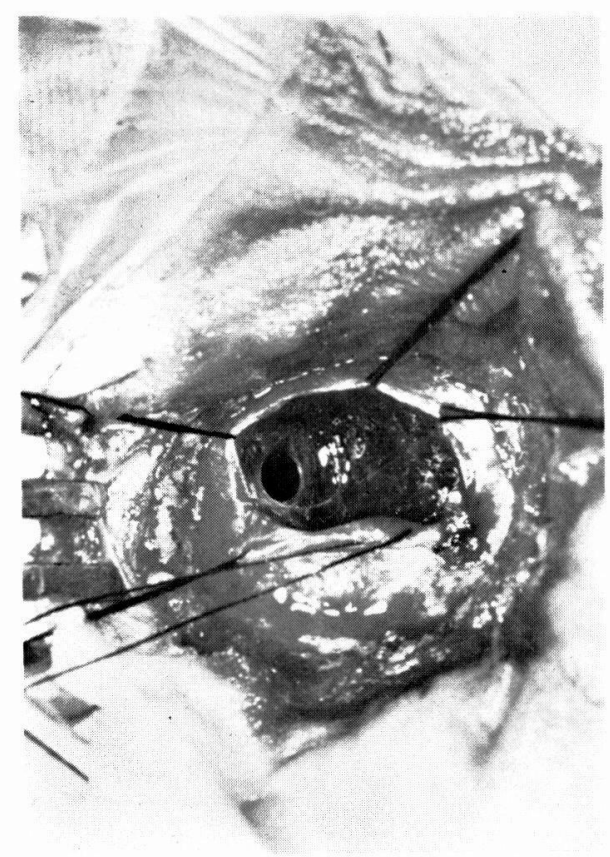

Fig. 5. Operative view of the cystic tumor in the posterior fossa. 

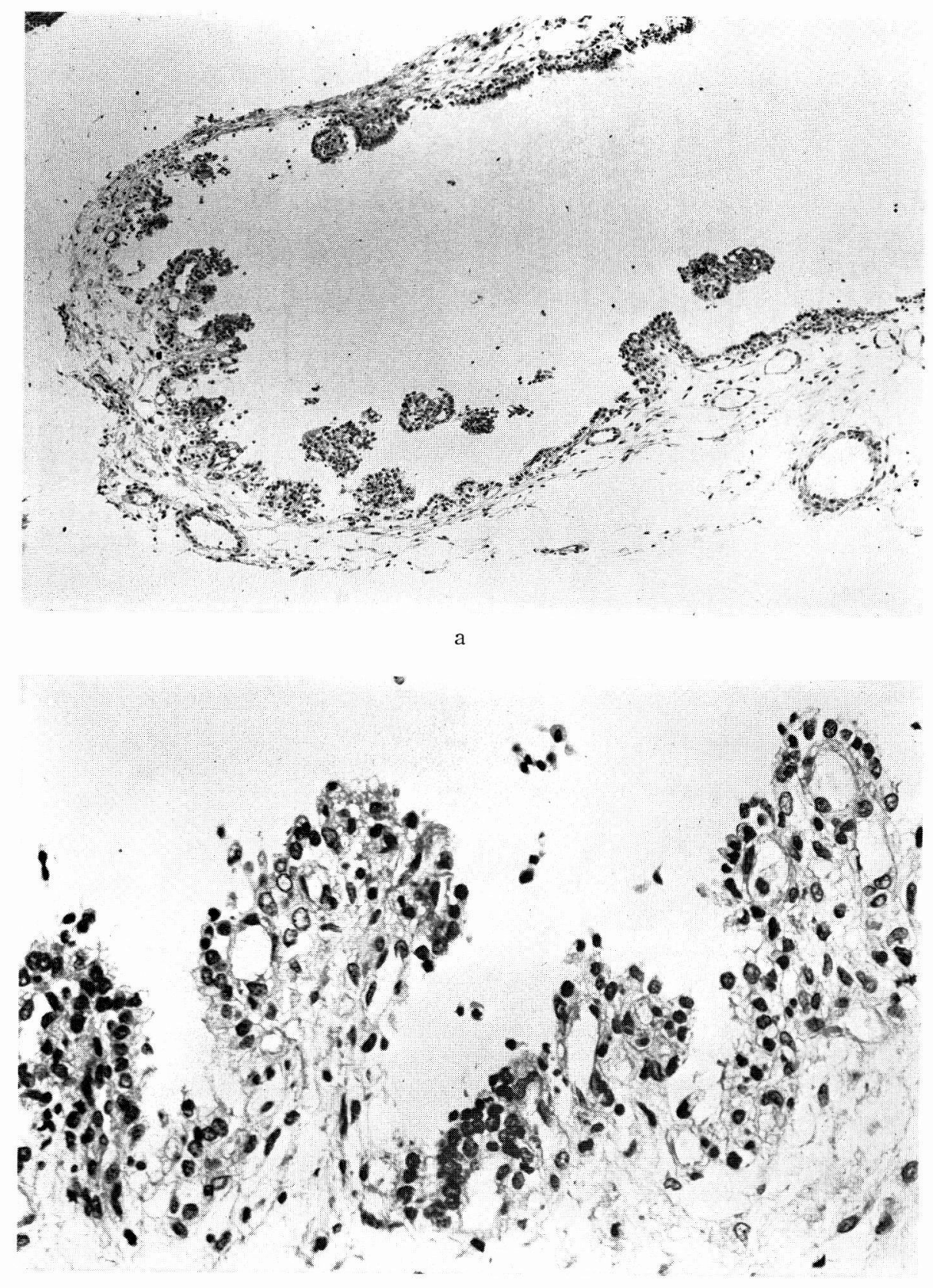

b

Fig. 6 .

a) Histology of the cyst wall showing neuroepithelial cyst with epithelial-lined tubule. $\times 240$

b) The cyst was lined internally by simple cuboidal and columnar epithelium. $\times 490(\mathrm{H}$ and $\mathrm{E})$ 
CT showed slight enlargement of the ventricles but no suspicion of recurrence in the supratentorial area. A circular, low density mass (cystic tumor), however, was observed in the cerebello-pontine angle. The forth ventricle was compressed and shifted slightly to the left. Metrizamide was injected into the reservoir of the right ventricle to confirm that there was no communication between the cyst and the ventricular system. Twenty days after hospitalization, an enlarged cystic tumor was clearly observed.

Clinically, new brain stem symptoms, such as stridor and nystagmus, appeared. Surgical operation was, therefore, performed five days later.

\section{Operative findings}

Craniectomy was performed in the prone position, with a paramedian incision of the posterior fossa. The cyst, covered by a brown membrane, was observed just below the opened dura mater. The fluid content was similar to that of the cerebrospinal fluid. The operation was completed after wide removal of the membrane (Fig. 5).

\section{Histological findings:}

The inner wall of the cyst was similar to the epithelial choroid plexus and covered with cuboidal epithelium. A part of the inner wall was directed toward the inner cavity and showed development of a papillary, and tubular formation. The epithelium was adjacent to the connective tissue just below it. PTAH staining did not show any basal corpuscle and other stainings (PAS, alcian blue) did not show any production of mucus (Fig. 6). Microscopically, the seeding (choroid plexus papilloma) could not be distinguished from the neuroepithelial cyst. Since the tumor developed only once and did not recur, even after five years, the tumor was considered to be a neuroepithelial cyst, which had developed in the posterior fossa.

The present case has survived since the operation for five years. At present, mental and physical growth are fairly normal, although the disturbance of the right oculomotor nerve remains.

\section{Discussion}

Since Maier (1861) first reported a neonatal brain tumor which developed in the lateral ventricle, the specificity of neonatal brain tumors has been regarded with interest. Arnstein (1975) defined a brain tumor that develops within sixty days after birth, as a neonatal brain tumor, whereas Jellinger (1973) reported that a brain tumor that develops even within one year after birth was possibly congenital. We believe that, because a neonatal brain tumor is relatively progressive and tends to become large, it exhibits clinical symptoms within a short period of time, even if it is not malignant, histologically. In this sense, we agree with the definition of Arnstein.

Sato et al. (1975) have reviewed a total of 154 cases of neonatal brain tumor. They state that, teratoma and tumor of neuroectodermal origin (medulloblastoma, ependymoma, astrocytoma, choroid plexus papilloma, etc.) occurred with the highest frequency. The former type was observed in sixty-two cases $(39 \%)$ and the latter type in sixty-three cases $(40 \%)$. The two kinds of brain tumor, therefore, made up about $80 \%$ of the total brain tumor cases. How ever, only nine cases, including the present case, of choroid plexus papilloma (one of neuroectodermal origin) have been reported (Table 2). This neonatal brain tumor developed in the right ventricle (six cases), the left ventricle (one case) and the third ventricle (the remaining two cases). No case of development in the posterior fossa has been reported (Table 2). Rovit (1970) reviewed a total of 234 cases of choroid plexus papilloma in all age groups. According to his report, $43 \%$ developed in the lateral ventricle and all these cases occur- 
TABLE 2

Choroid plexus papilloma in the newborn

\begin{tabular}{|c|c|c|c|c|c|c|c|}
\hline Case & author & & $\operatorname{sex}$ & $\begin{array}{l}\text { onset of initial } \\
\text { symptom }\end{array}$ & location & operation & outcome \\
\hline 1. & Wagenen & (1930) & - & $60 d$ ays & Lt. lateral Vent. & + & death \\
\hline 2 . & Drucker & (1939) & - & at birth & Rt. lateral Vent. & - & death \\
\hline 3 . & Matson & (1952) & $\mathrm{F}$ & 30 days & Rt. lateral Vent. & + & good \\
\hline 4. & Ray & (1956) & $\mathrm{F}$ & 60 days & Rt. lateral Vent. & + & death \\
\hline 5. & Palacios & (1962) & $\mathrm{F}$ & 40 days & Rt. lateral Vent. & - & death \\
\hline 6. & Cuatio & (1962) & M & 60 days & 3 rd. Vent. & + & death \\
\hline 7 . & Raskind & (1964) & $\mathrm{F}$ & 60 days & Rt. lateral Vent. & + & good \\
\hline 8. & Sato & (1975) & M & 60 days & 3 rd. Vent. & + & death \\
\hline 9 . & Honda & (1979) & $\mathrm{M}$ & 20 days & Rt. lateral Vent. & + & good \\
\hline
\end{tabular}

red in children aged less than ten years. A marked difference was observed before and after the age of ten, namely that as the age of patients increased, the incidence for the posterior fossa (the fourth ventricle) increased while the incidence for the lateral ventricle markedly decreased. In association with such a difference, in incidence, by age groups, Green (1951) proposed that a brain tumor did not always develop in the lateral ventricle itself and that it might develop in the embryonal rests, in other words, in the region adjacent to the lateral ventricle. However, there are still many problems to be solved in respect to the origin of development of brain tumors.

The overwhelming majority of the clinical symptoms was progressive hydrocepha lus (enlargement of cephalic circumference, enhancement of cephalic pressure) and other accompanying symptoms, such as, reduction of suckling ability, convulsions and disorders of conciousness were observed in most cases. However, local manifestations of involvement of the cranial nerves were hardly even observed. In fact, the present case showed only a disturbance of the right oculomotor nerve. The virtual lack of local symptoms appears to increase the difficulty of making an early diagnosis. Concerning the mechanism for the development of hy drocephalus due to choroid plexus papilloma, it is reported that excessive CSF secretion was observed in older children (Eisenberg et al. 1974 ; Fairburn, 1960) and that the narrow path for the cerebrospinal fluid was occluded directly by tumor (Bohm, 1961). In addition, Russell (1977) reported cases in which secondary diseases caused by tumor, such as ependymitis and basal meningitis, later became apparent. We believe that because neonatal tumor tends to become giant, occlusion of the path for the cerebrospinal fluid might be caused by direct compression due to tumor and secondary intraventricular hemorrhage due to tumor hemorrhage. The cerebrospinal fluid was contaminated with blood in the present case, as well as in Raskind's case (Raskind, 1964). Consideration of these observations suggests that the present case developed according to the latter mechanism.

Matson (1953) was the first to succeed in the overall extraction of neonatal choroid plexus papilloma by surgical treatment. This tumor developed in the right lateral ventricle of a four-weeks old girl. Thus, only three cases of the choroid plexus papil loma, including Raskind's and the present case, have been reported. The important 
factors for such aggravation of operative prognosis may be considered as follows: (1) the tumor tends to be giant. (2) subarachnoid hemorrhage (Abbott et al. 1957; Ernsting, 1955) and intraventricular hemorrhage may be easily caused because the tumor contains many blood vessels and ventricular drainage cannot effectively prevent the occlusion. (3) seeding occurs easily (Gillis, 1956; Beckett et al. 1950). (4) congenital abnormalities, such as Arnold-Chiari malformation, meningocele, etc., occur frequently as complications (Ovary, 1972; Russell, 1977). (5) the choroid plexus papilloma, which develops from the lateral ventricle, readily becomes malignant. From the operative aspect, it should be pointed out that confirmation of feeding artery and maintenance of humoral circulation are both difficult. In this context, more adequate maintenance of circulation is necessary. In future, the introduction of CT scanning may contribute to earlier and more accurate diagnosis of the tumor, especially in respect of its location, thereby, improving post-operative progress.

Stridor occurred in the present case about four months after excision of the papilloma. CT scan showed formation of a cystic tumor localized in the cerebello-pontine angle and the hemisphere of the cerebellum was compressed and shifted. As mentioned, histological distinction between the seeding (choroid plexus papilloma) and the neuroepithelial cyst was difficult. This is because, in many cases, the seeding tissue is very similar to the normal choroid plexus. Nevertheless, we considered the present case to be a neuroepithelial cyst on the basis of the following findings. (1) In most cases, seeding develops from the fourth ventricle and the seeding is diffuse morphologically and multiple nodules are easily formed (Raimondi, 1975) though in a few cases, a single nodule is formed. (2) Neonatal neuroepithelial cyst has been, although rarely, reported (Yoshida et al. 1983) and, in this case, the cyst developed from the subarachnoid cavity, deviating from the medianline. (3) No recurrence has been observed even more than five years after the operation.

Neuroepithelial cyst is sometimes called colloid cyst, ependymal cyst or choroid plexus cyst. The cyst develops frequently in the third ventricle (Shuangshoti et al. 1965). The cyst is also often observed in the ventricular system on the median line, including the fourth ventricle (Sharpe, 1977), the spinal tube (Morello, 1975; Mosso, 1975) and the quadrigeminal plate (Beckett et al. 1950). In some cases, the cyst develops in such areas, deviating from the median line, as the cerebellum (Hasegawa et al. 1976), the cerebral parenchyma (Friede, 1977; Ghatak et al. 1974) and the subarachnoid space (Jakubiak et al. 1968; Tandon et al. 1972).

Concerning the origin of development of the cyst, Kappers (1955) thought that the cyst was produced through the degeneration and separation of embryonic diencephalic vesicular recesses in the choroid fold and inferred that it might be the reason for its frequent occurrence in the third ventricle. Shuangshoti et al. (1965) proposed that the primitive neuroepithelium might develop from the neural tube in the fetal period, through evagination and invagination and emphasized its relation with the choroid plexus in the cyst.

Tandon et al. (1972) considered that the rare development of the cyst in the subarachnoid space and the cerebral parenchyma might be related to the heterotronic glial nests having the ependymal component in the leptomeninx, and Palma (1975) stated that development of the cyst in the cerebral parenchyma might be caused by primary epedymal ectopia.

Neuroepithelial cyst is said to have secretory ability (Ghatak et al. 1974). In the present case, CSF-like fluid erupted from the cyst. Other reports have described the inclusion of CSF lipid, PAS-resctive material (Shuangshoti et al. 1965), mucicarmin 
(Ghatak et al. 1974). The cyst is enlarged by the secretory action and exhibits clinical symptoms when it becomes 1 to 3 $\mathrm{cm}$ in length. A very frequent sign is increased intracranial pressure (hydrocephalus). In addition, when the cyst develops in the subarachnoid cavity of the posterior fossa, as in the present case, the brain stem is compressed and such clinical symptoms as stridor, hemiparesis and nystagmus appear.

Most patients develop the cyst at the age of twenty to thirty years, more commonly in females. In infants, the cyst rarely occurs and is localized, if at all, at the region deviating from the median line or outside the ventricles. Histologically, the cyst has a structure similar to the choroid plexus and ependyma and is in the form of monolayer covered with cuboidal or columnar epithelium, while tubular and papillary formation is observed in part. The epithelium is adjacent to the connective tissue. In the present case, mucus production was not detected by PAS or alcian blue staining. However, in some cases, mucus secreting cells were detected. In some cases, a cilial character has been observed as in the ependyma.

\section{Conclusion}

We have succeeded in the total removal of a neonatal choroid plexus papilloma in the right lateral ventricle. No similar case has been reported in this country. We consider the present case to be extremely in teresting, because the neuroepithelial cyst, which developed in the posterior fossa after the first operation, was closely related to the choroid plexus papilloma, from both histological and embryological aspects.

\section{Reference}

Abbott, K., Rollas, Z. H. and Meagher, J. N.
(1957). Choroid plexus papilloma causing spontaneous subarachnoid hemorrhage. Report of case and review of literatur. J. Neurosurg. 14, 566-570.

Arnstein, L. H., Boldrey, E. and Nafzinger, H. C. (1951). A case report and surgery of brain tumors during the neonatal period. J. Neurosurg. 8, 315-319.

BeCKeTt, R. S., Netsky, M. C. and Zimmerman, H. M. (1950). Developmental stenosis of the aqueduct of Sylvius. Am. J. Pathol. 26, 755-771.

Bohm, E. and Strang, R. (1961). Choroid plexus papilloma. J. Neurosurg. 18, 493-500.

Cautio, G. and Castan, P. (1962). Choroid plexus papilloma of third ventricle. J. Neurosurg. 40, $1085-1087$.

Drucker, G. A. (1939). Papillary tumor of the choroid plexus in a newborn infant. Arch Path. 28, 390-395.

Eisenberg, H. M., McComb, J. G. and Lorezo, A. (1974). Cerebrospinal fluid overproduction and hydrocephalus associated with choroid plexus papilloma. J. Neurosurg. 40, 381-385.

ERnsting, J. (1955). Choroid plexus papilloma causing spontaneous subarachnoid hemorrhage. J. Neurol Neurosurg Psychiat. 18, 134-136.

Fairburn, B. (1960). Choroid plexus papilloma and its relation to hydrocephalus. J. Neurosurg. 17, 166-171.

Friede, R. L. and YASARGiL, M. G. (1977). Supratentorial intracerebral epithelial (ependymal) cysts-review, case reports, and fine structure. J. Neurol Neurosurg Psychiatry. 40, 127-137.

Gardner, W. J. and TuRner, O. A. (1938). Neuroepithelial cyst of third ventricle-report of case with recovery following operation. Arch Neurol Psychiat. 38, 1055.

Ghatak, N. R., Hirano, A., Kasoff, S. S. and ZiMMERMAN, H. M. (1974). Fine structure of an intracerebral epithelial cyst. J. Neurosurg. 41, 75-82.

Gillis, D. A. and DiProfio, C. R. F. (1956). Papillary tumors of the choroid plexus. Cand. Med. Ass. J. 74, 361-363.

GREENE, R. C. (1951). Extraventricular and intracerebellar papilloma of the choroid plexus. J. Neuropath Exp Neurol. 10, 204-207.

Hall, G. W. and Fentress, T. L. (1933). Papilloma choroideum with diffuse central nervous system metastasis J. Neurol psychopath. 14, 108.

Hasegawa, H., Ushio, Y., Oku, Y., Iwata, Y., 
Kanai, N. and Kamikawa, K. (1976). Neuroepithelial cyst of the cerebellar vermis. Surg Neurol. 6, 181-184.

Jakubiak, P., Dunsmore, R. H. and Backett, R. S. (1968). Supratentorial brain cysts. J. Neurosurg. 28, 129-136.

Jellinger, K. and Plassmann, M. S. (1973). Congenital intractranial tumors. Neuropediatrie. 4, 46-63.

KAPPERS, J. A. (1955). Development of paraphysis cerebri in man with comments on its relationship to intercolumnar tubercle and its significance for origin of cystic tumors in third ventricle. J. Comp Neurol. 102, 425.

MaIER, R. (1861). Combinierte Geschwulst im Grosshirn. Virchows Arch. Path. Anat. Physiol. $20,536-541$.

Matson, D. D. (1953). Hydrocephalus in a premature infant caused by papilloma of the choroid plexus with report of surgical treatment. J. Neurosurg. 10, 416-420.

Morello, G. and Lombardi, G. (1975). Choroidoepedymal cysts of the spinal roots. Case report. J. Neurosurg. 21,1103-1107.

Mosso, J. A. and Verity, M. A. (1975). Ependymal cyst of the spinal cord. Case report J. Neurosurg. 43, 757-760.

Ovary, E. (1972). Connatal brain tumors. Donausymp. Neurophath. Wien 1971 Wien Klin Wschr. 84, 433.

Palacios, E. and Lawson, R. C. (1972). Choroid plexus papillomas of the lateral ventricles. Amer. J. Roentgen. 115, 113-119.

Palma, L. (1975). Supratentorial neuroepithelial cysts. Report of two cases. J. Neurosurg. 42, 353-357.

Raimondi, A. J. and Gutierrez, F. A. (1975). Di agnosis and surgical treatment of choroid plexus papilloma. Child's Brain. 1, 81-115.

RASKIND, R. and BELGeL, F. (1964). Brain tumors in early infancy-probably congenital in origin.
J. Pediat. 65, 727-732.

RAY, B. S. and PECK, R. C. (1956). Papilloma of the choroid plexus of the lateral ventricles causing hydrocephalus in an infant. J. Neurosurg. 13, 317-322.

Rovit, R. L., Schechter, M. M. and Chodroff, P. (1970). Observation on radiographic diagnosis. Amer. J. Roentogen. 110, 608-617.

Russell, D. S. and Rubinstein, L. J. (1977). Pathology of tumor of the central nervous system. London, Arnold pp. 220-224.

Sato, N. and Sakamoto, K. (1978). Treatment cases of brain tumor of early infants. Child's Brain, 1, 121-125.

Sharpe, J. A. and Deck, J. H. N. (1977). Neuroepithelial cyst of the fourth ventricle. J. Neurosurg. 46, 820-824.

ShuAngshoti, S., Roberts, M. P. and Netsky, M. G. (1965). Neuroepithelial (colloid) cysts. Pathogenesis and relation to choroid plexus and ependyma Arch Pathol. 80, 214-224.

STоокеу, B. J. (1934). Intermittent obsturction of foramen of Monro by neuroepithelial cyst of third ventricle. Bull Neur Inst New York. $3,446$.

Tandon, P. N., Roy, S. and Elvidge, A. (1972). Subarachnoid ependymal cyst. Report of two cases. J. Neurosurg. 37, 741-745.

WAGENEN, W. P. (1930). Papilloma of the choroid Plexus. Report of two cases, one with removal of tumor at operation, and one with "seeding" of tumor in the ventricular system. Arch Surg. 20, 199-231.

Wolfson, W. L. and Brown, W. L. (1977). Choroid plexus papilloma. An ultrastructural study. Arch Pathol Lab Med. 101, 366-368.

Yoshida, T., Nakayani, S., Ushio, Y., Hayakawa, T. and Mogami, H. (1983). Huge epithelial lined cyst in infancy. Neurol Med Chir (Tokyo). 23, 393-397. 